\title{
Programmed task based motion analysis of robotic systems equipped with flexible links and supports
}

\author{
Elżbieta Jarzębowska ${ }^{1 *}$, Krzysztof Augustynek ${ }^{2}$, and Andrzej Urbaś ${ }^{2}$ \\ ${ }^{1}$ Warsaw University of Technology, 00-665 Warsaw, Nowowiejska 24, Poland \\ ${ }^{2}$ University of Bielsko-Biala, 43-309 Bielsko-Biala, Willowa 2, Poland
}

\begin{abstract}
The automatic computational procedure to derivate dynamics equations of systems with programmed constraints modified to encompass compliant mechanical components in their structures is discussed in the paper. The dynamics analysis of the compliant manipulator model with a flexible link is presented as an example. The rigid finite element method is used in order to take into account the flexibility of the link. The formalism of joint coordinates and homogeneous transformations are used to describe the manipulator motion.
\end{abstract}

\section{Introduction}

Analysis of robot task based motions dedicated to work and services delivery is presented in the paper. The key contribution of the paper is in three aspects of the analysis. The first one is the possibility of constraints incorporation into the system dynamics, the second one is the special computational procedure for the constrained dynamics generation, which provides dynamic models, referred to as reference dynamics, satisfying all constraints upon them. The third contribution comparing to the results reported in the literature, see e.g. $[1,2]$ is in modeling flexibility of the system parts and supports. The constraints put on a system are referred to as programmed and they are imposed as control goals on system performance or service task requirements. The procedure of generating reference dynamics offers automated generation of equations and it was successfully developed and implemented to rigid system models [3, 4]. The advantage of this procedure is that it serves both reference and control oriented dynamics derivation and the final dynamics models are obtained in the reduced state form, i.e. constraint reaction forces are eliminated. The procedure is extended on flexible subsystems of lightweight and fast machine parts and servicing equipment, which are prone to vibration in some work regimes. Vibrations may significantly affect system performance and disable effective controller designs. Flexibility of links is modeled using the rigid finite element method, whose advantage relies in its ability of application of the rigid body approach to modeling flexible link elements. The novelty of the presented method is its ability to analyze any system reference motion, including flexible link vibration, in the presence of kinematic task-based constraints. The results of this analysis can contribute to verification of a system behavior when it is subjected to given kinematic constraints, help to specify desired task-based constraints properly, e.g. put tighter velocity limits, to exclude some work regimes or to design controllers correctly. The possibility of reference motion analysis in these aspects enables simulation of various work regimes for system models and selection of required and safe task based motion parameters, accordingly. The theoretical development is illustrated by simulation studies of an example of a flexible link and support manipulator model, whose service tasks are predefined by the programmed constraints. Special interest of this study is to analyze flexible link vibrations when the manipulator performs the tasks.

\section{Mathematical model of a manipulator}

Figure 1 presents a compliant supported manipulator model. It is assumed, that the manipulator is driven by means of a driving torque $\mathbf{t}_{d r}^{(2)}$. The manipulator third link is flexible. The rigid finite element method is used to discretize this link.

The following joint coordinates are used to describe motion of the manipulator:

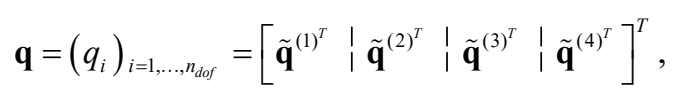

where: $\tilde{\mathbf{q}}^{(1)}=\left[z^{(1)}\right], \tilde{\mathbf{q}}^{(2)}=\left[\psi^{(2)}\right]$,

\footnotetext{
${ }^{*}$ Corresponding author: elajarz@meil.pw.edu.pl
} 


$$
\begin{aligned}
& \tilde{\mathbf{q}}^{(3)}=\left[\begin{array}{lllll}
\tilde{\mathbf{q}}^{(3,0)^{T}} & \cdots & \tilde{\mathbf{q}}^{(3, r)^{T}} & \cdots & \tilde{\mathbf{q}}^{\left(3, n_{r f e}^{(3)}\right)^{T}}
\end{array}\right]^{T}, \\
& \tilde{\mathbf{q}}^{(3,0)}=\left[\psi^{(3,0)}\right], \tilde{\mathbf{q}}^{(3, r)}=\left[\begin{array}{lll}
\psi^{(3, r)} & \theta^{(3, r)} & \varphi^{(3, r)}
\end{array}\right]^{T}, \\
& \tilde{\mathbf{q}}^{(4)}=\left[z^{(4)}\right] \text {. }
\end{aligned}
$$

Vector $\mathbf{q}$ contains independent and dependent coordinates:

$$
\mathbf{q}=\left(q_{i}\right)_{i \in i_{i c} \cup i_{d_{c}}} \in\left\{\mathbf{q}_{i_{c}}, \mathbf{q}_{d_{c}}\right\},
$$

where: $i_{i_{c}} \in\left\{1,2,4, \ldots, n_{d o f-1}\right\}$ and $i_{d_{c}} \in\left\{3, \ldots, n_{d o f}\right\}$ are independent and dependent coordinates indexes.

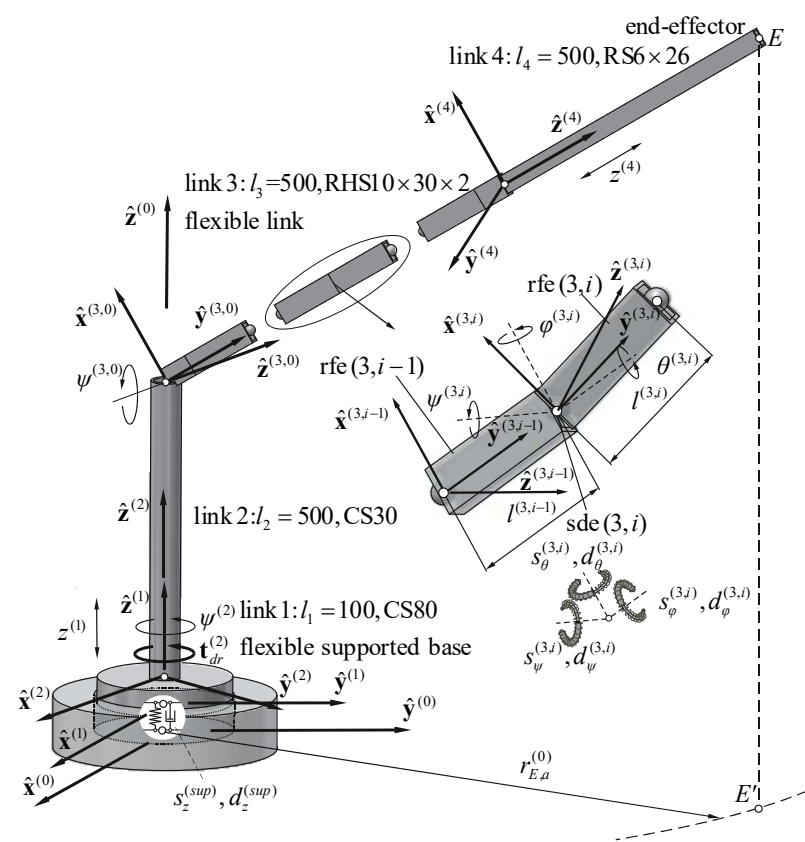

Figure 1. Model of a manipulator.

The generalized programmed motions equations (GPME) with first order programmed constraints underlie the derivation procedure and they have the form $[3,4]$ :

$$
\left.\frac{\partial R_{1}}{\partial \dot{q}_{i}}\right|_{i \in i_{i_{c}}}+\sum_{j \in i_{d_{c}}} \frac{\partial R_{1}}{\partial \dot{q}_{j}} \frac{\partial \dot{q}_{j}}{\partial \dot{q}_{i}}=0
$$

where:

$$
\begin{aligned}
& R_{1}=\dot{E}_{k}-2 \sum_{i=1}^{n_{\text {dof }}} \frac{\partial E_{k}}{\partial q_{i}} \dot{q}_{i}+\sum_{i=1}^{n_{\text {dof }}} \frac{\partial E_{p}}{\partial q_{i}} \dot{q}_{i}+\sum_{i=1}^{n_{\text {dof }}} \frac{\partial R_{s}}{\partial \dot{q}_{i}} \dot{q}_{i}-\sum_{i=1}^{n_{\text {dof }}} t_{i} \dot{q}_{i}, \\
& E_{k}=\sum_{l=1}^{n_{l}} E_{k}^{(l)}, E_{k}^{(l)}=\frac{1}{2} \operatorname{tr}\left\{\dot{\mathbf{T}}^{(l)} \mathbf{H}^{(l)}\left(\dot{\mathbf{T}}^{(l)}\right)^{T}\right\}, \\
& E_{p}=E_{p, g}+E_{p, \text { sup }}+E_{p, f_{l}}, E_{p, g}=\sum_{l=1}^{n_{l}} E_{p, g}^{(l)}, \\
& E_{p, g}^{(l)}=m^{(l)} g \mathbf{J}_{3} \mathbf{T}^{(l)} \mathbf{r}_{C^{(l)}}^{(l)}, \mathbf{J}=\left[\begin{array}{l}
\mathbf{J}_{1} \\
\mathbf{J}_{2} \\
\mathbf{J}_{3}
\end{array}\right]=\left[\begin{array}{llll}
1 & 0 & 0 & 0 \\
0 & 1 & 0 & 0 \\
0 & 0 & 1 & 0
\end{array}\right], \\
& E_{p, \text { sup }}=\frac{1}{2} s_{z}^{(\text {sup })}\left(z^{(1)}\right)^{2},
\end{aligned}
$$

$$
\begin{aligned}
& E_{p, f_{l}}=\frac{1}{2} \sum_{s=1}^{n_{s e}^{(l)}}\left(\tilde{\mathbf{q}}^{(l, s)}\right)^{T} \mathbf{S}^{(l, s)} \tilde{\mathbf{q}}^{(l, s)}, \mathbf{S}^{(l, s)}=\operatorname{diag}\left\{s_{\psi}^{(l, s)}, s_{\theta}^{(l, s)}, s_{\varphi}^{(l, s)}\right\}, \\
& R_{s}=R_{\text {sup }}+R_{f_{l}}, R_{\text {sup }}=\frac{1}{2} d_{z}^{(s u p)}\left(\dot{z}^{(1)}\right)^{2}, \\
& R_{f_{l}}=\frac{1}{2} \sum_{s=1}^{n_{s e}^{(l)}}\left(\dot{\tilde{\mathbf{q}}}^{(l, s)}\right)^{T} \mathbf{D}^{(l, s)} \dot{\tilde{\mathbf{q}}}^{(l, s)}, \mathbf{D}^{(l, s)}=\operatorname{diag}\left\{d_{\psi}^{(l, s)}, d_{\theta}^{(l, s)}, d_{\varphi}^{(l, s)}\right\}, \\
& \mathbf{t}=\left[\begin{array}{lll}
0 & t_{d r}^{(2)} & \mathbf{0}
\end{array}\right]^{T} .
\end{aligned}
$$

It is assumed that the end-effector moves along an elliptical trajectory designed in a plane parallel to the $x^{(0)} y^{(0)}$ plane and its $z_{E}^{(0)}$ coordinate has to change according to assumed time-dependent function $z_{E, a}^{(0)}(t)$. Thus, these programmed constraints can be written in the form:

$$
\begin{aligned}
& \Phi_{1} \equiv 0 \Rightarrow\left(\frac{x_{E}^{(0)}}{a_{E, a}^{(0)}}\right)^{2}+\left(\frac{y_{E}^{(0)}}{b_{E, a}^{(0)}}\right)^{2}-1=0 \\
& \Phi_{2} \equiv 0 \Rightarrow z_{E}^{(0)}-z_{E, a}^{(0)}(t)=0
\end{aligned}
$$

where: $x_{E}^{(0)}=\mathbf{J}_{1} \mathbf{T}^{(4)} \mathbf{r}_{E}^{(4)} y_{E}^{(0)}=\mathbf{J}_{2} \mathbf{T}^{(4)} \mathbf{r}_{E}^{(4)} z_{E}^{(0)}=\mathbf{J}_{3} \mathbf{T}^{(4)} \mathbf{r}_{E}^{(4)}$, According to the GPME algorithm [3], the constraint equations are differentiated to obtain

$$
\begin{aligned}
& \dot{\Phi}_{1} \equiv 0 \Rightarrow \mathbf{u} \dot{\mathbf{q}}=\mathbf{0}, \\
& \dot{\Phi}_{2} \equiv 0 \Rightarrow \mathbf{C}_{3} \dot{\mathbf{q}}-\dot{z}_{E, a}^{(0)}(t)=0, \\
& \ddot{\Phi}_{1} \equiv 0 \Rightarrow \mathbf{u} \ddot{\mathbf{q}}+v=0, \\
& \ddot{\Phi}_{2} \equiv 0 \Rightarrow \mathbf{C}_{3} \ddot{\mathbf{q}}+d_{3}-\ddot{z}_{E, a}^{(0)}(t)=0,
\end{aligned}
$$

with:

$$
\begin{aligned}
& \mathbf{C}=\left[\begin{array}{l}
\mathbf{C}_{1} \\
\mathbf{C}_{2} \\
\mathbf{C}_{3}
\end{array}\right]=\left(c_{i j}\right)_{\substack{i=1,2,3 \\
j=1, \ldots, 4}}=\mathbf{J}\left[\begin{array}{lll}
\mathbf{T}_{1}^{(4)} \mathbf{r}_{E}^{(4)} & \cdots & \mathbf{T}_{4}^{(4)} \mathbf{r}_{E}^{(4)}
\end{array}\right], \\
& \mathbf{d}=\left(d_{i}\right)_{i=1, \ldots, 3}=\mathbf{J}\left(\left(\sum_{i=1}^{n_{\text {dof }}} \sum_{j=1}^{n_{\text {dof }}} \mathbf{T}_{i j}^{(4)} \dot{q}_{i} \dot{q}_{j}\right) \mathbf{r}_{E}^{(4)}\right) \\
& \mathbf{u}=\left(u_{j}\right)_{j=1, \ldots, 4}=\frac{1}{\left(a_{E, a}^{(0)}\right)^{2}} \mathbf{J}_{1} \mathbf{T}^{(4)} \mathbf{r}_{E}^{(4)} \mathbf{C}_{1}+\frac{1}{\left(b_{E, a}^{(0)}\right)^{2}} \mathbf{J}_{2} \mathbf{T}^{(4)} \mathbf{r}_{E}^{(4)} \mathbf{C}_{2}, \\
& v=\frac{1}{\left(a_{E, a}^{(0)}\right)^{2}}\left(\left(\mathbf{C}_{1} \dot{\mathbf{q}}\right)^{2}+\mathbf{J}_{1} \mathbf{T}^{(4)} \mathbf{r}_{E}^{(4)} d_{1}\right)+ \\
& +\frac{1}{\left(b_{E, a}^{(0)}\right)^{2}}\left(\left(\mathbf{C}_{2} \dot{\mathbf{q}}\right)^{2}+\mathbf{J}_{2} \mathbf{T}^{(4)} \mathbf{r}_{E}^{(4)} d_{2}\right),
\end{aligned}
$$

$a_{E, a}^{(0)}, b_{E, a}^{(0)}$ are the elliptical trajectory semi-major and semi-minor axes, respectively.

Vector of dependent velocities are as follows:

$\dot{\mathbf{q}}_{d_{c}}=-\mathbf{K}_{d_{c}}^{-1} \mathbf{K}_{i_{c}} \dot{\mathbf{q}}_{i_{c}}$,

where:

$$
\mathbf{K}_{d_{c}}=\left[\begin{array}{ll}
u_{2} & u_{4} \\
c_{12} & c_{14}
\end{array}\right], \mathbf{K}_{i_{c}}=\left[\begin{array}{ll}
u_{1} & u_{3} \\
c_{11} & c_{13}
\end{array}\right] .
$$

The GPME equations supplemented by the equations of programmed constraints take the form: 


$$
\begin{aligned}
& {\left[\begin{array}{c}
\left.\mathbf{M}_{i}\right|_{i \in i_{i_{c}}}+\sum_{j \in i_{d_{c}}} \mathbf{M}_{j} \frac{\partial \dot{q}_{j}}{\partial \dot{q}_{i}} \\
\mathbf{u} \\
\mathbf{C}_{3}
\end{array}\right] \ddot{\mathbf{q}}=} \\
& =\left[\begin{array}{c}
\mathbf{h}_{i}+\mathbf{Q}_{i}+\sum_{j \in i_{i_{c}} \cup i_{d_{c}}} \dot{q}_{j} \frac{\partial Q_{j}}{\partial \dot{q}_{i}}+\ldots \\
\ldots \sum_{k \in i_{d_{c}}}\left(h_{k}+Q_{k}+\sum_{j \in i_{i c} \cup i_{i_{c}}} \dot{q}_{j} \frac{\partial Q_{j}}{\partial \dot{q}_{k}}\right) \frac{\partial \dot{q}_{k}}{\partial \dot{q}_{i}} \\
-v \\
-d_{3}+\ddot{z}_{E, a}^{(0)}(t)
\end{array}\right],
\end{aligned}
$$

where:

$$
\begin{aligned}
& \mathbf{M}=\sum_{l=1}^{n_{l}} \mathbf{M}^{(l)}, \mathbf{M}^{(l)}=\left(m_{i j}^{(l)}\right)_{i, j=1, \ldots, n_{d o f}^{(l)}}, \\
& m_{i j}^{(l)}=\operatorname{tr}\left\{\mathbf{T}_{i}^{(l)} \mathbf{H}^{(l)}\left(\mathbf{T}_{j}^{(l)}\right)^{T}\right\}, \\
& \mathbf{h}=\sum_{l=1}^{n_{l}} \mathbf{h}^{(l)}, \mathbf{h}^{(l)}=\left(h_{i}^{(l)}\right)_{i=1, \ldots, n_{d o f}^{(l)}}, \\
& h_{i}^{(l)}=\sum_{m=1}^{n_{a l o l}^{(l)}} \sum_{n=1}^{(l)}\left(\operatorname{tr}\left\{\mathbf{T}_{m}^{(l)} \mathbf{H}^{(l)}\left(\mathbf{T}_{m, n}^{(l)}\right)^{T}\right\} \dot{q}_{m}^{(l)} \dot{q}_{n}^{(l)}+\right. \\
& +2 \sum_{m=1}^{n_{\text {dof }}^{(l)}} \sum_{n=1}^{n_{\text {lof }}^{(l)}} \operatorname{tr}\left\{\mathbf{T}_{m}^{(l)} \mathbf{H}^{(l)}\left(\mathbf{T}_{i, n}^{(l)}\right)^{T}\right\} \dot{q}_{m}^{(l)} \dot{q}_{n}^{(l)}, \\
& \mathbf{T}_{i}^{(l)}=\frac{\partial \mathbf{T}^{(l)}}{\partial q_{i}^{(l)}}, \mathbf{T}_{i, j}^{(l)}=\frac{\partial^{2} \mathbf{T}^{(l)}}{\partial q_{i}^{(l)} \partial q_{j}^{(l)}}, \\
& \mathbf{Q}=-(\mathbf{g}+\mathbf{d}+\mathbf{f})+\mathbf{t}, \\
& \mathbf{g}=\sum_{l=1}^{n_{l}} \mathbf{g}^{(l)}, \mathbf{g}^{(l)}=\left(g_{i}^{(l)}\right)_{i=1, \ldots, n_{\text {alof }}^{(l)}}, g_{i}^{(l)}=m^{(l)} g \mathbf{J}_{1} \mathbf{T}_{i}^{(l)} \mathbf{r}_{C^{(l)}}^{(l)}, \\
& \mathbf{f}=\left[\begin{array}{llll}
f^{(1)} & 0 & \mathbf{f}^{(3)} & 0
\end{array}\right]^{T}, \\
& f^{(1)}=s_{z}^{(s u p)} z^{(1)}, \mathbf{f}^{(3)}=\left[\begin{array}{llll}
0 & \mathbf{S}^{(3,1)} \tilde{\mathbf{q}}^{(3,1)} & \ldots & \mathbf{S}^{\left(3, n_{s d d}^{(3)}\right)} \tilde{\mathbf{q}}^{\left(3, n_{s d e}^{(3)}\right)}
\end{array}\right], \\
& \mathbf{d}=\left[\begin{array}{llll}
d^{(1)} & 0 & \mathbf{d}^{(3)} & 0
\end{array}\right]^{T}, \\
& d^{(1)}=d_{z}^{(s u p)} z^{(1)}, \mathbf{d}^{(3)}=\left[\begin{array}{llll}
0 & \mathbf{D}^{(3,1)} \dot{\tilde{\mathbf{q}}}^{(3,1)} & \ldots & \mathbf{D}^{\left(3, n_{s d e}^{(3)}\right.} \dot{\tilde{\mathbf{q}}}^{\left(3, n_{s d e}^{(3)}\right)}
\end{array}\right],
\end{aligned}
$$

H - pseudo-inertia matrix, $g$ - the acceleration of gravity.

\section{Simulation study - programmed motion of the crane end-effector}

Simulation studies are carried out for two cases: the manipulator with rigid links and flexible support and the manipulator with the flexible link and flexible support. The following parameters are used in simulation: $s_{z}^{(s u p)}=10^{4} \mathrm{Nm}^{-1}, \quad d_{z}^{(s u p)}=2.5 \mathrm{Nsm}^{-1}, \quad a_{E, a}^{(0)}=0.375 \mathrm{~m}$,
$b_{E, a}^{(0)}=0.750 \mathrm{~m}$. It is assumed that the end-effector $z_{E}^{(0)}$ coordinate changes according to the following function:

$z_{E, a}^{(0)}(t)=z_{0}+A \cos \left(\frac{2 \pi}{T} t\right)$,

where $z_{0}$ is the initial position of the end-effector, $A$ is the amplitude and $T$ is a time period of oscillations. It is assumed that $A=0.5 \mathrm{~m}$ and $T=2.5 \mathrm{~s}$. The column (2) is driven by the driving torque defined by:

$$
t_{d r}^{(1)}(t)= \begin{cases}10 t_{d r, 0}^{(1)}\left(\frac{t}{t_{0}}\right)^{3}-15 t_{d r, 0}^{(1)}\left(\frac{t}{t_{0}}\right)^{4}+6 t_{d r, 0}^{(1)}\left(\frac{t}{t_{0}}\right)^{5}, & t<t_{0}, \\ t_{d r, 0}^{(1)}, & t \geq t_{0} .\end{cases}
$$

It is assumed $t_{0}=5 \mathrm{~s}$ and $t_{d r, 0}^{(1)}=1 \mathrm{Nm}$.

The dynamic equations of motion are integrated using $4^{\text {th }}$ order Runge-Kutta scheme with using the Baumgarte stabilization method for the programmed constraint equations. Figure 2 shows time courses of the joint coordinates obtained from simulations.
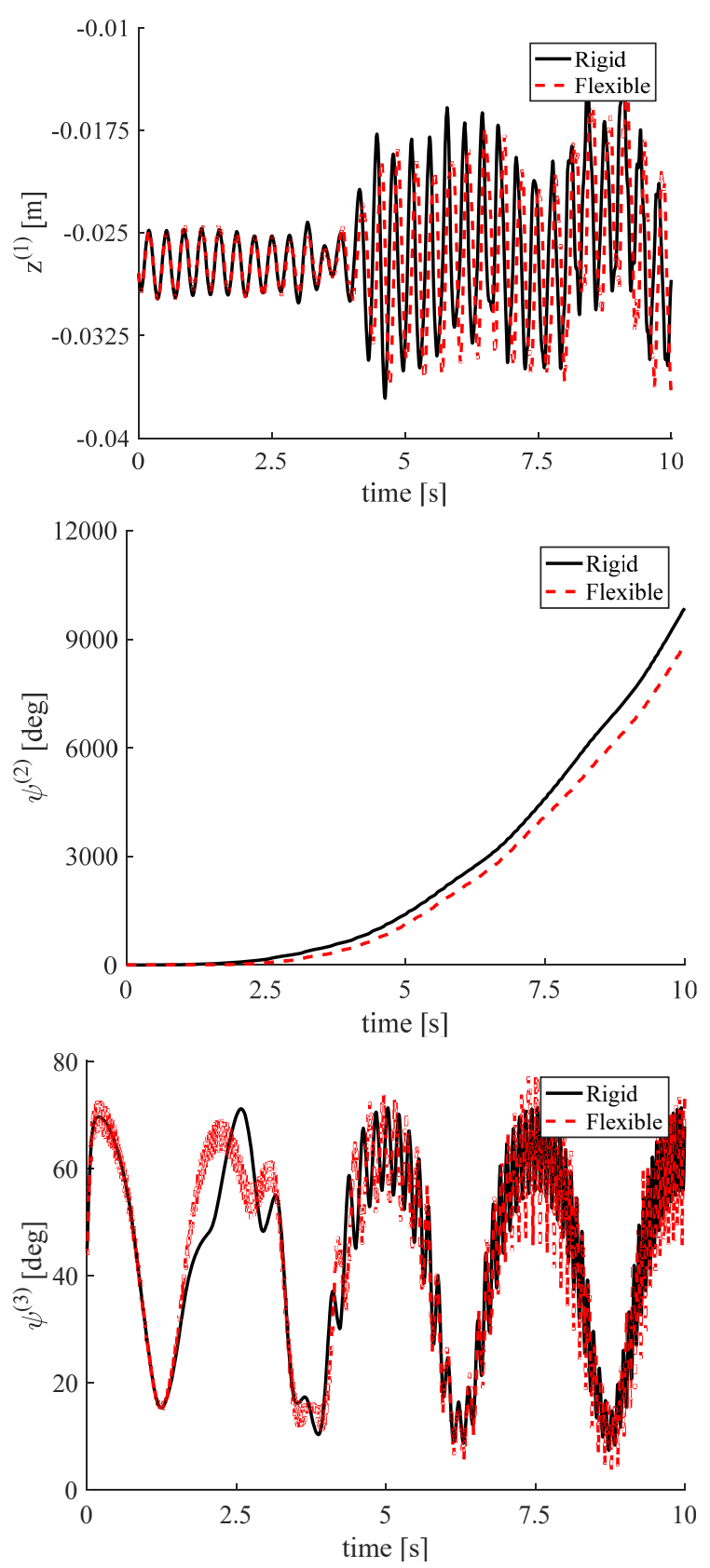


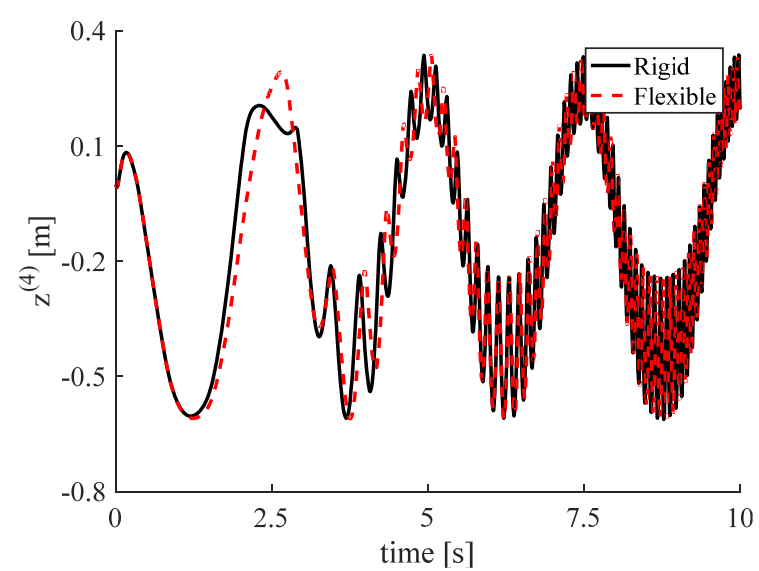

Figure 2. Time course of the joint coordinates

It can be observed that the link flexibility has significant influence on motion of the manipulator. The results show that the programmed motion of the manipulator is compensated by the appropriate combination of the rotation angle of the arm 3 and the displacement of the slider 4. When the flexibility is taken into account additional vibrations with small amplitude and large frequency are induced at initial phase of the motion. After $5 \mathrm{~s}$, the amplitude of vibrations of arm 3 is larger than those obtained for the rigid body model.

Figure 3 presents the course of programmed constraints.
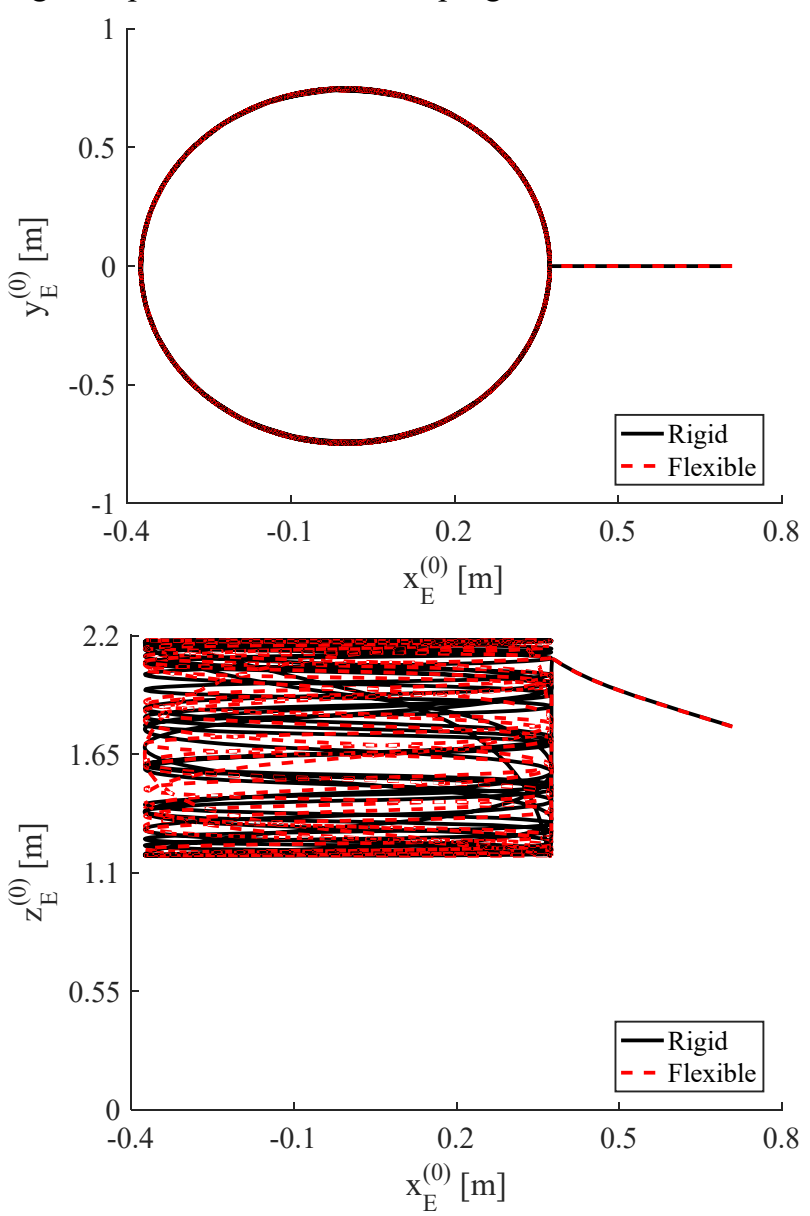

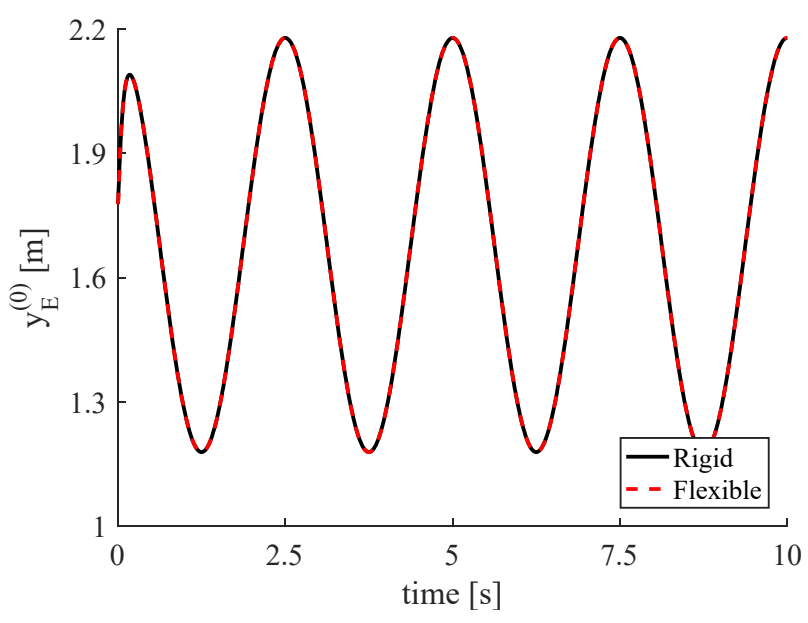

Figure 3. The end-effector trajectory and the time course of $z_{E}^{(0)}$ coordinate

It can be observed a good agreement of the obtained courses with the assumed programmed constraints. At the initial phase of motion the end-effector is located outside the assumed trajectory and after some period of time this trajectory is achieved.

\section{Conclusions}

The computational reference model of a crane equipped with the flexible link and support subjected to first order programmed constraints is presented. It is generated by the automatic computational algorithm designed using the formalism of joint coordinates and homogeneous transformations. The algorithm enables constraints incorporation into the system dynamics and modeling flexibility of the system parts and supports. The presented approach can be generalized for systems with higher order constraints.

\section{References}

1. A.A. Ata, H. Johar, International Journal of Advanced Robotic Systems, 1, 2 (2004)

2. A.A. Ataa, W.F. Faresb, M.Y. Sa'adehc, Procedia Engineering, 41 (2012)

3. E. Jarzębowska, K. Augustynek, A. Urbaś, Concurrency and Computation: Practice and Experience (2018), doi:10.1002/cpe.4452. 25

4. E. Jarzębowska, K. Augustynek, A. Urbaś, Proc. of ASME Design Eng. Techn. Conf., No UNSP V006T10A016 (2017) 